\title{
Avaliação da qualidade de amostras comerciais de leite de janaguba (Himatanthus drasticus (Mart.) Plumel) em Fortaleza - Ceará
}

SOARES, F.P. ${ }^{*}$; ALMEIDA, F.S.1; MIRANDA, C.C. ${ }^{1 ;}$ CARVALHO, P.H.L.1; ROMERO, N.R.2; BANDEIRA, M.A.M. ${ }^{2}$ 1 Universidade de Fortaleza, Av. Washington Soares, 1321, CEP: 60811-905, Fortaleza-Brasil. 2Universidade Federal do Ceará, R. Capitão Francisco Pedro, 1210, CEP: 60430-370, Fortaleza-Brasil. *Autor para correspondência: fabiana@unifor.br

RESUMO: Os fitoterápicos à base de leite de janaguba (Himatanthus drasticus (Mart.) Plumel), usados para o tratamento de câncer, úlcera gástrica e outras doenças, são muito vendidos em mercados públicos de Fortaleza (CE). No entanto, registros mencionam que é comum a troca deste leite por látex de mangabeira (Hancornia speciosa Gomes). O trabalho objetivou avaliar a qualidade físico-química, química e microbiológica de amostras comerciais do leite de janaguba. Dez amostras comerciais foram adquiridas de um mercado de Fortaleza; quatro amostras autênticas de látex de janaguba foram obtidas da chapada do Araripe e uma amostra de látex de mangabeira foi obtida em Paracuru (CE). Foram determinados o aspecto geral, densidade, $\mathrm{pH}$, resíduo seco, volume de sedimentação, perfil cromatográfico e qualidade microbiológica das amostras. Os resultados mostraram elevada contaminação microbiológica nas preparações comerciais e adulteração em seis destas amostras, o que aponta a urgente implantação de uma efetiva farmacovigilância dos fitoterápicos a fim de tornar seu consumo mais seguro e racional.

Palavras-chave: fitoterápicos, qualidade, látex; janaguba; Himatanthus drasticus

\begin{abstract}
Quality assessment of commercial samples of janaguba's milk (Himatanthus drasticus (Mart.) Plumel) in Fortaleza - Ceará. The herbal medicines made from janaguba milk (Himatanthus drasticus (Mart.) Plumel), used to treat gastric ulcer, cancer and other diseases, and arewidely sold in the public markets of Fortaleza (CE). However, records mention that it is common to make fake copies of the milk by using mango tree latex (Hanconia speciosa Gomes). This study aimed to evaluate the chemical, physical-chemical and microbiological quality of commercial samples of janaguba milk. Ten commercial samples were purchased from a Fortaleza market; four authentic samples of janaguba latex were obtained from the Araripe plateau, and one mango tree latex sample was obtained in Paracuru (CE). We determined the general appearance, density, $\mathrm{pH}$, dry residue, sedimentation volume, chromatographic profile and microbiological qualities. Results showed high microbiological contamination in the commercial preparations and evidence of tampering in six of these samples, which indicates the need for an urgent implementation of an effective pharmacovigilance strategy for herbal medicines in order to make its consumption more secure and rational.
\end{abstract}

Key words: herbal medicines, quality, latex; janaguba; Himatanthus drasticus

\section{INTRODUÇÃO}

A fitoterapia é o tratamento de enfermidades utilizando plantas medicinais. Seu uso seguro e racional deve ser buscado por profissionais de saúde e por usuários, porém a automedicação é um fato frequente devido ao seu fácil acesso. Isso é preocupante, pois a população está exposta a vários riscos devido à má utilização das plantas medicinais (Simões et al., 2001).

Nas últimas décadas, tem havido uma tendência mundial de aumento na demanda por fitoterápicos, influenciada por fatores econômicos, sociais e culturais. Este aumento associado à falta de fiscalização efetiva, que garanta desde a exploração racional dos recursos naturais empregados como matéria-prima, até chegar ao produto acabado, contribui para a disponibilidade e acesso a produtos, muitas vezes sem condições adequadas ao uso, sem garantia da qualidade, segurança e eficácia ou efetividade fundamentais para recuperação e manutenção da saúde do paciente (Souza \& Maciel, 
2010).

Com o crescimento da produção de fitoterápicos, aumenta também a dificuldade em se fazer o controle de qualidade desses produtos. Este controle é dividido em várias etapas, começando com a obtenção da matéria-prima, finalizando com a análise do produto acabado (Nascimento et al., 2005). A preocupação com a qualidade desses fitoterápicos tem aumentado e raizeiros e ervanários deveriam atender a critérios de adequação a saúde para garantir ao consumidor um produto seguro. A má qualidade das plantas medicinais e a farmacovigilância incipiente são os principais fatores observados que fazem com que este problema se agrave (Freitas, 2009).

Para manter a qualidade de um fitoterápico são necessárias práticas adequadas de identificação, cultivo, coleta, beneficiamento, armazenamento e transporte dos mesmos, o que não acontece com produtos comercializados por raizeiros (Marcondes \& Esmerino, 2010). Dentre as várias preparações fitoterápicas comercializadas em ervanários e feiras livres do Ceará, encontram-se aquelas à base de látex de Himatanthus drasticus (Mart.) Plumel (Apocynaceae), conhecidas popularmente como leite de janaguba (Lorenzi \& Matos, 2008). Esta preparação é usada empiricamente para o tratamento do câncer, vermes intestinais, febre, menstruações irregulares, infertilidade feminina, sintomas da menopausa e úlceras gástricas (Lorenzi \& Matos, 2008; Lucetti et al., 2010; Mousinho at al., 2011; Oliveira et al., 2012). Estudos farmacológicos revelaram efeitos antiúlcera, antitumoral, imunomodulador, analgésico e antiinflamatório, que em parte justificam alguns dos usos populares (Lucetti et al., 2010; Colares et al., 2008a; Colares et al., 2008b; Leite et al., 2009; Sousa, 2009).

Segundo Peixoto (2001), há registros de que os índios Cariris utilizavam a janaguba existente na chapada do Araripe, no início da colonização, como cicatrizante. Em 1976, o médico cearense José Ulisses Peixoto afirmou que o látex de janaguba possuía alto poder imunológico contra alguns tipos de câncer. A declaração era de um conceituado clínico do Cariri, que se baseava em estudos procedidos ao longo de quatro anos acompanhando de perto pacientes cancerosos que foram tratados, consumindo ou aplicando, o leite de janaguba. Apesar de muitos questionamentos em torno da real eficácia, o fato é que litros com o leite diluído em água são vendidos para todo o país e para o exterior (Peixoto, 2001).

Lorenzi \& Matos (2008) relatam que é comum a troca do leite de janaguba por leite de mangabeira (Hancornia speciosa Gomes; Apocynaceae), outra preparação de uso tradicional na região como medicação contra tuberculose. Assim, diante da possibilidade de troca de derivados vegetais no preparo de leite de janaguba, bem como a qualidade dessas preparações, o presente trabalho objetivou avaliar a qualidade físico-química, química e microbiológica de amostras comerciais do leite de janaguba.

\section{MATERIAL E MÉTODO}

\section{Amostras}

Dez amostras comerciais de leite de janaguba (codificadas de $\mathrm{A}$ a J) foram adquiridas de um mercado de Fortaleza, CE, onde são comercializadas por raizeiros. Foram obtidas durante os meses de maio a junho de 2011 (A a E) e março de 2012 (F a J). A Tabela 1 apresenta a origem, embalagem, data de fabricação e tipos de informações presentes nas rotulagens de cada uma delas.

Para fins comparativos foram utilizados os parâmetros físico-químicos e químicos determinados para quatro amostras autênticas de látex de $H$. drasticus (LJ1 a LJ4) coletadas na Chapada do Araripe (Crato, CE) por um extrativista florestal cadastrado pelo IBAMA (Instituto Brasileiro do Meio Ambiente e dos Recursos Naturais Renováveis). Segundo relato do extrativista, faz-se raspagem suave nos caules da planta, colhe-se o látex com uma colher e armazena-se em recipiente higienizado (látex bruto). Para comercializar, o látex bruto é diluído em água filtrada e fervida na proporção de 25 partes de látex para 75 de água $(1: 4, v / v)$. No comércio é determinada a validade de quatro meses, a contar da data de extração, sob refrigeração. As amostras de látex na forma de suspensão aquosa 1:4 (v/v) foram adquiridas em períodos distintos (novembro de 2009, julho de 2011, fevereiro de 2012 e novembro de 2012).

Amostras das plantas foram identificadas como Himatanthus drasticus por comparação com exsicata depositada no Herbário Prisco Bezerra da Universidade Federal do Ceará (UFC), Fortaleza, CE, sob o número 31685. Das espécies de Himatanthus que ocorrem no Brasil, $H$. drasticus é a única encontrada no Ceará (Plumel, 1990; Forzza et al., 2010), predominando na Chapada do Araripe (Modesto, 1997; Amaro et al., 2006).

Uma amostra de látex de caule de mangabeira (Hancornia speciosa) $(5,0 \mathrm{~mL})$ foi diluída em $15,0 \mathrm{~mL}$ de água destilada, submetida a partição em acetato de etila $(4 \times 5,0 \mathrm{~mL})$ e o solvente evaporado por pressão reduzida em aparelho rotaevaporador. O resíduo extrativo foi denominado LM e utilizado no teste de cromatografia em camada delgada (CCD). Este látex foi coletado

Rev. Bras. PI. Med., Campinas, v.18, n.2, p.399-407, 2016. 
TABELA 1. Origem, embalagem, data de fabricação e tipos de informações presentes nas rotulagens das amostras comerciais de leite de janaguba.

\begin{tabular}{|c|c|c|c|c|}
\hline Amostra & Origem & Embalagem & $\begin{array}{c}\text { Data de } \\
\text { fabricação }\end{array}$ & Tipo de informações na rotulagem \\
\hline A & Chorozinho* & $\begin{array}{l}\text { Garrafa de vidro transparente }(1 \mathrm{~L}) \\
\text { com vedação de plástico. }\end{array}$ & Maio/2011* & Não possui rotulagem \\
\hline B & Crato* & $\begin{array}{l}\text { Garrafa de vidro transparente }(1 \mathrm{~L}) \\
\text { com vedação de plástico. }\end{array}$ & Maio/2011* & Nome comercial; indicação terapêutica \\
\hline C & Cascavel* $^{*}$ & $\begin{array}{l}\text { Garrafa de vidro transparente }(1 \mathrm{~L}) \\
\text { com vedação de plástico. }\end{array}$ & Abril/2011 & $\begin{array}{l}\text { Nome comercial; indicação terapêutica; modo de usar; data } \\
\text { de fabricação; validade ( } 6 \text { meses) }\end{array}$ \\
\hline D & Crato & $\begin{array}{l}\text { Garrafa de vidro transparente (1 L) } \\
\text { com vedação de cortiça. }\end{array}$ & Junho/2011* & $\begin{array}{l}\text { Nome comercial; indicação terapêutica; conservação; modo } \\
\text { de usar; endereço do fabricante }\end{array}$ \\
\hline E & Crato* & $\begin{array}{l}\text { Garrafa de vidro transparente }(1 \mathrm{~L}) \\
\text { com vedação de cortiça. }\end{array}$ & Junho/2011* & Nome comercial; indicação terapêutica; modo de usar \\
\hline $\mathbf{F}$ & Crato* $^{*}$ & $\begin{array}{l}\text { Garrafa de vidro transparente }(1 \mathrm{~L}) \\
\text { com vedação de plástico. }\end{array}$ & Março/2012* & Nome comercial; indicação terapêutica; modo de usar \\
\hline G & Crato* & $\begin{array}{l}\text { Garrafa de vidro transparente }(1 \mathrm{~L}) \\
\text { com vedação de plástico. }\end{array}$ & Março/2012* & Nome comercial; indicação terapêutica \\
\hline $\mathbf{H}$ & Crato* & $\begin{array}{l}\text { Garrafa de vidro transparente }(1 \mathrm{~L}) \\
\text { com vedação de plástico. }\end{array}$ & Março/2012* & Nome comercial; indicação terapêutica; validade (6 meses) \\
\hline $\mathbf{I}$ & Crato & $\begin{array}{l}\text { Garrafa de vidro transparente }(1 \mathrm{~L}) \\
\text { com vedação de cortiça. }\end{array}$ & Março/2012* & $\begin{array}{l}\text { Nome comercial; indicação terapêutica; conservação; modo } \\
\text { de usar; endereço do fabricante }\end{array}$ \\
\hline $\mathbf{J}$ & Crato & $\begin{array}{l}\text { Garrafa de vidro transparente }(1 \mathrm{~L}) \\
\text { com vedação de cortiça. }\end{array}$ & Março/2012* & $\begin{array}{l}\text { Nome comercial; indicação terapêutica; modo de usar; } \\
\text { endereço do fabricante }\end{array}$ \\
\hline
\end{tabular}

*Provável origem e/ou data de fabricação.

em um sítio localizado em Paracuru (CE), em março de 2012, e exemplares das partes aéreas da planta foram identificadas por comparação com amostra depositada no Herbário Prisco Bezerra da UFC, sob o número 48849.

Todas as amostras foram analisadas nos períodos em que foram adquiridas.

\section{Avaliação do aspecto geral das amostras}

Volumes de 2,0 mL das amostras de leite de janaguba foram transferidos para tubos de ensaio para determinação das características organolépticas e aspecto visual (homogeneidade e consistência). A cor foi determinada após prévia agitação manual dos tubos.

\section{seco}

Determinação de $\mathrm{pH}$, densidade e resíduo

Foram realizados em triplicata e conforme metodologia descrita na Farmacopeia Brasileira (2011).

\section{Determinação do volume de sedimentação} Esta metodologia foi feita conforme Gabriels \& Plaizier-Vercammen (2004), com modificações. Para estudar a sedimentação das suspensões, o volume de sedimentação foi determinado em função do tempo. Este teste é utilizado para suspensões mantidas em uma condição estacionária, onde as partículas sedimentam somente no sentido vertical devido à ação da força da gravidade (Shojaeia \& Arefinia, 2006).

As amostras foram transferidas para provetas de $10,0 \mathrm{~mL}$ e deixadas em repouso para decantação da suspensão. A determinação foi feita em triplicata.

Foram feitas duas leituras de sedimentação: a primeira em 1 hora, e a segunda em 24 h. O volume de sedimentação $F$ é definido como a relação do volume final do sedimento em equilíbrio (Vf) com o volume total da suspensão (Vt), tal como expresso na seguinte equação: $F=[V f / V t]$. Este parâmetro é utilizado para verificar a estabilidade física da suspensão, sendo que quanto maior for o valor da razão $F$ mais elevado é o grau de floculação (Lachman et al., 2001).

etila

\section{Substâncias extraíveis por acetato de}

A Farmacopeia Brasileira (2010) descreve o método para determinação de substâncias extraíveis por álcool, sugerindo o uso de outros solventes desde que especificados em monografia. H. drasticus não é uma planta oficial, e neste caso, optou-se por usar o acetato de etila (AcOEt) por se constituir em solvente orgânico com polaridade intermediária, possibilitando a realização de partição do látex.

Para cada uma das amostras de leite de janaguba foi separado um volume de $100,0 \mathrm{~mL}$ 
que foi submetido à extração por partição com o solvente AcOEt. Foram realizadas cinco extrações com volumes de $25,0 \mathrm{~mL}$ cada. Houve formação de uma fase intermediaria, sendo esta separada em um béquer e posteriormente submetida a lavagens com AcOEt (10 x 10,0 mL).

A fase orgânica foi reunida com as extrações obtidas pela partição da amostra. Os solventes foram evaporados em banho-maria $\left(50^{\circ} \mathrm{C}\right)$ e $\mathrm{o}$ resíduo extrativo, pesado em balança analítica.

\section{Tratamento estatístico dos dados analíticos}

Os resultados das análises físico-químicas foram expressos calculando-se a média aritmética de cada determinação (triplicata), sendo a amplitude de variação em torno da média determinada através do cálculo do desvio padrão (D.P.) e em torno de várias médias, pelo erro padrão da média (E.P.M.) (Leite, 1998).

\section{Análise por Cromatografia em Camada Delgada (CCD)}

Foram preparadas soluções em acetato de etila nas concentrações de $5,0 \mathrm{mg} / \mathrm{mL}$ a partir dos resíduos extrativos em acetato de etila resultantes da partição com as amostras, totalizando 10 soluções-amostras para a CCD (A a J). O mesmo procedimento foi realizado com o resíduo extrativo em acetato de etila de LJ (LJ1 a LJ4) e do látex de mangabeira (LM).

Foram empregadas placas pré-elaboradas de gel de sílica de 12,0×11,5 cm (cromatofolha Merck, gel de sílica 60 F254, 0,2 mm) e para fase móvel foi utilizado o solvente orgânico clorofórmio de pureza analítica. A revelação foi realizada pelo método físico da luz ultravioleta (254 nm) e emprego de vapores de iodo (Soares, 2015).

\section{Qualidade microbiológica}

A verificação da qualidade microbiológica foi realizada conforme metodologia descrita na Farmacopeia Brasileira (2010). A contagem de micro-organismos viáveis totais foi realizada pelo método da semeadura em profundidade ou Pour Plate. As análises das amostras de leite de janaguba não ocorreram simultaneamente, porém foram descritas em conjunto.

\section{RESULTADO E DISCUSSÃO}

\section{Aspecto geral das amostras}

As amostras de referência LJ (LJ1 a LJ4) apresentaram coloração bege leitosa escura, odor forte e ácido e aspecto visual bifásico e fluido.

Observou-se que as amostras A, B, C, F, G e H são, de uma forma geral, distintas de D, E, I e J. As amostras B e G, pelo odor forte e fétido, podem estar com a qualidade microbiológica claramente comprometida. As amostras D, E, I e J apresentaram aspecto visual e odor compatíveis com LJ, porém a cor apresentou-se mais clara, provavelmente devido a diferenças na diluição do látex nas preparações. A Tabela 2 apresenta os resultados da avaliação do aspecto geral das amostras comerciais.

\section{seco}

\section{Determinação de $\mathrm{pH}$, densidade e resíduo}

$\mathrm{O}$ resultado das determinações de $\mathrm{pH}$, densidade e resíduo seco das quatro amostras de referência LJ foram respectivamente: $5,30 \pm 0,02$; $1,0032 \pm 0,0005 \mathrm{~g} / \mathrm{mL} ;$ e $1,27 \pm 0,04 \%$ (p/v) (Tabela 3).

$\mathrm{O} \mathrm{pH}$ ácido para a suspensão aquosa 1:4 (v/v) de látex de janaguba foi também observado para o látex bruto de $H$. succuba, porém com valor distinto, 4,54 (Silva et al., 2003). Para o látex bruto de mangabeira, Pinheiro et al. (2004) citam valores de $\mathrm{pH}$ mais ácidos, em torno de 3,8 .

As amostras B e $\mathrm{G}$ apresentaram pHs diferentes de LJ, sendo a primeira mais ácida e a segunda, neutra. As densidades de C, D e E apresentaram valores de aproximadamente $50 \%$ das demais amostras, inclusive de LJ. Os resultados de resíduo seco também foram variados, sendo que

TABELA 2. Aspecto geral das amostras comerciais de leite de janaguba.

\begin{tabular}{cccl}
\hline Amostra & Cor & Odor & Aspecto visual \\
\hline A & Branco leitoso claro & Fraco & Homogêneo e fluido, mas com presença de partículas estranhas na superfície \\
B & Branco leitoso claro & Forte e fétido & Homogêneo e fluido, mas com presença de partículas estranhas na superfície \\
C & Branco leitoso claro & Fraco & Homogêneo e fluido, mas com presença de partículas estranhas na superfície \\
D & Bege leitoso & Forte e ácido & Bifásico e fluido \\
E & Bege leitoso & Forte e ácido & Bifásico e fluido \\
F & Branco leitoso claro & Fraco & Homogêneo e fluido, mas com presença de partículas estranhas na superfície \\
G & Branco leitoso claro & Forte e fétido & Homogêneo e fluido, mas com presença de partículas estranhas na superfície \\
H & Branco leitoso claro & Fraco & Homogêneo e fluido, mas com presença de partículas estranhas na superfície \\
I & Bege leitoso & Forte e ácido & Bifásico e fluido \\
J & Bege leitoso & Forte e ácido & Bifásico e fluido \\
\hline LJ & Bege leitoso escuro & Forte e ácido & Bifásico e fluido \\
\hline
\end{tabular}

${ }^{*}$ Características das amostras LJ1 a LJ4.

Rev. Bras. Pl. Med., Campinas, v.18, n.2, p.399-407, 2016. 
TABELA 3. Resultados de pH, densidade e resíduo seco das amostras autênticas de látex de $H$. drasticus.

\begin{tabular}{llll}
\hline Amostra & pH & Densidade $(\mathbf{g} / \mathbf{m L})$ & Resíduo seco $(\%, \mathbf{p} / \mathbf{v})$ \\
\hline LJ1 & $5,01 \pm 0,08$ & $1,0041 \pm 0,0022$ & $1,26 \pm 0,02$ \\
LJ2 & $5,96 \pm 0,02$ & $1,0058 \pm 0,0002$ & $1,28 \pm 0,01$ \\
LJ3 & $4,67 \pm 0,07$ & $1,0004 \pm 0,0008$ & $1,27 \pm 0,13$ \\
LJ4 & $5,57 \pm 0,00$ & $1,0024 \pm 0,0005$ & $1,29 \pm 0,14$ \\
\hline Média \pm E.P.M. & $5,30 \pm 0,02$ & $1,0032 \pm 0,0005$ & $1,27 \pm 0,04$ \\
\hline
\end{tabular}

apenas as amostras D e E apresentaram valores equivalentes a LJ. Tais variações podem estar relacionadas às diferentes diluições do látex nas preparações comerciais. A Tabela 4 apresenta os resultados das determinações de $\mathrm{pH}$, densidade e resíduo seco das amostras $\mathrm{A}$ a $\mathrm{J}$.

\section{Volume de sedimentação}

O resultado da avaliação de volume de sedimentação das amostras de referência

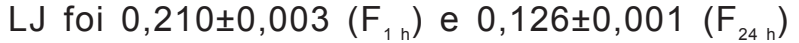
(Tabela 5). Através dos resultados de volume de sedimentação, observou-se que LJ tem o sedimento compactado em 24 horas de repouso, ou seja, há redução do volume de sedimentação em cerca de $40,0 \%$. Tal fato pode estar relacionado ao tamanho das partículas em suspensão: quanto maiores forem estas partículas, maior será sua sedimentação.

Comportamento contrário ocorreu com as amostras D, E, I e J, cujo sedimento aumentou em 24 horas e com valores distintos entre si (Tabela 6). Provavelmente estas amostras possuem partículas com tamanhos menores que as presentes em LJ, permanecendo suspensas por mais tempo. As demais amostras praticamente não apresentaram sedimento.

\section{Rendimento extrativo em acetato de etila}

O rendimento extrativo em AcOEt obtido por partição líquido-líquido das amostras de referência LJ foi de $0,68 \pm 0,02 \%$ (Tabela 7 ).

TABELA 4. $\mathrm{pH}$, densidade e resíduo seco das amostras comerciais de leite de janaguba.

\begin{tabular}{cccc}
\hline Amostra & $\mathrm{pH}$ & Densidade $(\mathrm{g} / \mathrm{mL})$ & Resíduo seco $(\%)$ \\
\hline A & $4,96 \pm 0,06$ & $1,0012 \pm 0,0000$ & $0,33 \pm 0,01$ \\
B & $3,61 \pm 0,01$ & $1,0016 \pm 0,0002$ & $0,38 \pm 0,03$ \\
C & $4,98 \pm 0,07$ & $0,5024 \pm 0,0022$ & $0,45 \pm 0,02$ \\
D & $4,82 \pm 0,15$ & $0,5018 \pm 0,0001$ & $1,32 \pm 0,04$ \\
E & $4,87 \pm 0,12$ & $0,5022 \pm 0,0006$ & $1,17 \pm 0,09$ \\
F & $5,11 \pm 0,02$ & $1,0013 \pm 0,0040$ & $0,25 \pm 0,04$ \\
G & $7,18 \pm 0,04$ & $1,0005 \pm 0,0014$ & $0,33 \pm 0,02$ \\
H & $4,95 \pm 0,03$ & $1,0001 \pm 0,0004$ & $0,37 \pm 0,02$ \\
I & $5,77 \pm 0,05$ & $1,0038 \pm 0,0004$ & $1,73 \pm 0,08$ \\
J & $4,96 \pm 0,02$ & $1,0053 \pm 0,0008$ & $2,75 \pm 0,18$ \\
\hline LJ & $5,30 \pm 0,02$ & $1,0032 \pm 0,0005$ & $1,27 \pm 0,04$ \\
\hline
\end{tabular}

TABELA 5. Resultado da avaliação de volume de sedimentação das amostras autênticas de látex de H. drasticus.

\begin{tabular}{ccc}
\hline Amostra & $\mathbf{F}_{1 \mathrm{~h}}{ }^{*}$ & $\mathbf{F}_{24 \mathrm{~h}}{ }^{*}$ \\
\hline LJ1 & $0,203 \pm 0,006$ & $0,120 \pm 0,000$ \\
LJ2 & $0,217 \pm 0,006$ & $0,127 \pm 0,006$ \\
LJ3 & $0,220 \pm 0,010$ & $0,137 \pm 0,006$ \\
LJ4 & $0,200 \pm 0,000$ & $0,120 \pm 0,000$ \\
\hline MédiatE.P.M. & $0,210 \pm 0,003$ & $0,126 \pm 0,001$ \\
\hline
\end{tabular}

${ }^{*} \mathrm{~F}_{1 \mathrm{~h}}$ e $\mathrm{F}_{24 \mathrm{~h}}$ : Volume de sedimento em 1 hora e em 24 horas, respectivamente. 
TABELA 6. Resultado da avaliação de volume de sedimentação das amostras comerciais de leite de janaguba em 1 hora e em 24 horas.

\begin{tabular}{ccc}
\hline Amostra & $\mathbf{F}_{1 \mathrm{~h}}$ & $\mathbf{F}_{24 \mathrm{~h}}$ \\
\hline A & $0,01 \pm 0,00$ & $0,01 \pm 0,00$ \\
B & $0,01 \pm 0,00$ & $0,01 \pm 0,00$ \\
C & $0,00 \pm 0,00$ & $0,00 \pm 0,00$ \\
D & $0,02 \pm 0,00$ & $0,09 \pm 0,01$ \\
E & $0,01 \pm 0,00$ & $0,06 \pm 0,00$ \\
F & $0,00 \pm 0,00$ & $0,01 \pm 0,00$ \\
G & $0,00 \pm 0,00$ & $0,00 \pm 0,00$ \\
H & $0,00 \pm 0,00$ & $0,01 \pm 0,00$ \\
I & $0,00 \pm 0,00$ & $0,05 \pm 0,01$ \\
J & $0,03 \pm 0,01$ & $0,12 \pm 0,06$ \\
\hline LJ & $0,21 \pm 0,00$ & $0,13 \pm 0,00$ \\
\hline
\end{tabular}

TABELA 7. Rendimento em substâncias extraíveis por acetato de etila, obtido por partição do látex de H. drasticus 1:4 (v/v).

\begin{tabular}{cc}
\hline Amostra & Rendimento $(\%)$ \\
\hline LJ1 & 0,68 \\
LJ2 & 0,66 \\
LJ3 & 0,67 \\
LJ4 & 0,70 \\
\hline Média \pm D.P. & $0,68 \pm 0,02$
\end{tabular}

A Tabela 8 apresenta a quantidade (massa em gramas) do conteúdo solúvel em acetato de etila extraído das amostras. Os rendimentos das amostras são variáveis e distintos de LJ. Figura 1

TABELA 8. Rendimento das frações em acetato de etila obtidas a partir das amostras comerciais e de referência de leite de janaguba.

\begin{tabular}{cc}
\hline Amostra & Rendimento (\%) \\
\hline A & 0,05 \\
B & 0,16 \\
C & 0,11 \\
D & 0,59 \\
E & 0,78 \\
F & 0,45 \\
G & 0,32 \\
H & 0,33 \\
I & 0,54 \\
J & 0,50 \\
\hline LJ & $0,68 \pm 0,02$ \\
\hline
\end{tabular}

\section{Cromatografia em camada delgada}

A CCD revelou que as amostras LJ apresentam perfil cromatográfico semelhante, com três manchas principais com $R_{\mathrm{fs}}$ aproximadamente de $0,13,0,54$ e 0,64. A mancha com $R_{f} 0,64$ apresenta fluorescência quando observada sob radiação ultravioleta ( $\Lambda 254 \mathrm{~nm}$ ).

As amostras D, E, I e $\mathrm{J}$ correspondem à janaguba por apresentarem perfil cromatográfico semelhante. Por outro lado, as amostras A, B, C, F e $G$ apresentaram um perfil distinto, porém idêntico ao do látex de mangabeira (LM), com duas manchas principais com $R_{t s}$ aproximadamente de 0,18 e 0,46. $A$ amostra $H$ apresentou manchas com $R_{\mathrm{fs}}$ de LJ e LM, podendo se tratar de uma mistura de látex das duas plantas. A Figura 1 apresenta as imagens do perfil cromatográfico das amostras analisadas reveladas com vapores de iodo.

Apenas $40,0 \%$ das amostras ensaiadas correspondem à amostra autêntica de $\mathrm{H}$. drasticus. Visualmente é fácil distinguir a preparação autêntica de adulterada: o leite de janaguba apresenta um decantado bege e um sobrenadante límpido castanho-claro; enquanto o leite de mangabeira se apresenta como uma emulsão branca, sem decantado no fundo da garrafa. A substituição ou mistura com látex de mangabeira (Hancornia speciosa) pode estar relacionada a ocorrência comum da planta no Ceará, bem como em outros estados brasileiros (Aguiar Filho et al., 1998), constituindo-se em planta laticífera de fácil acesso. Por outro lado, é pouco provável que haja confusão na identificação botânica das espécies, pois são plantas bastante distintas pela morfologia das folhas, flores e frutos (Sampaio, 2008).

\section{Qualidade microbiológica}

Com relação à qualidade microbiológica das amostras LJ, os resultados se encontraram no limite das especificações da Farmacopeia Brasileira (2010). Todas foram negativas para presença 

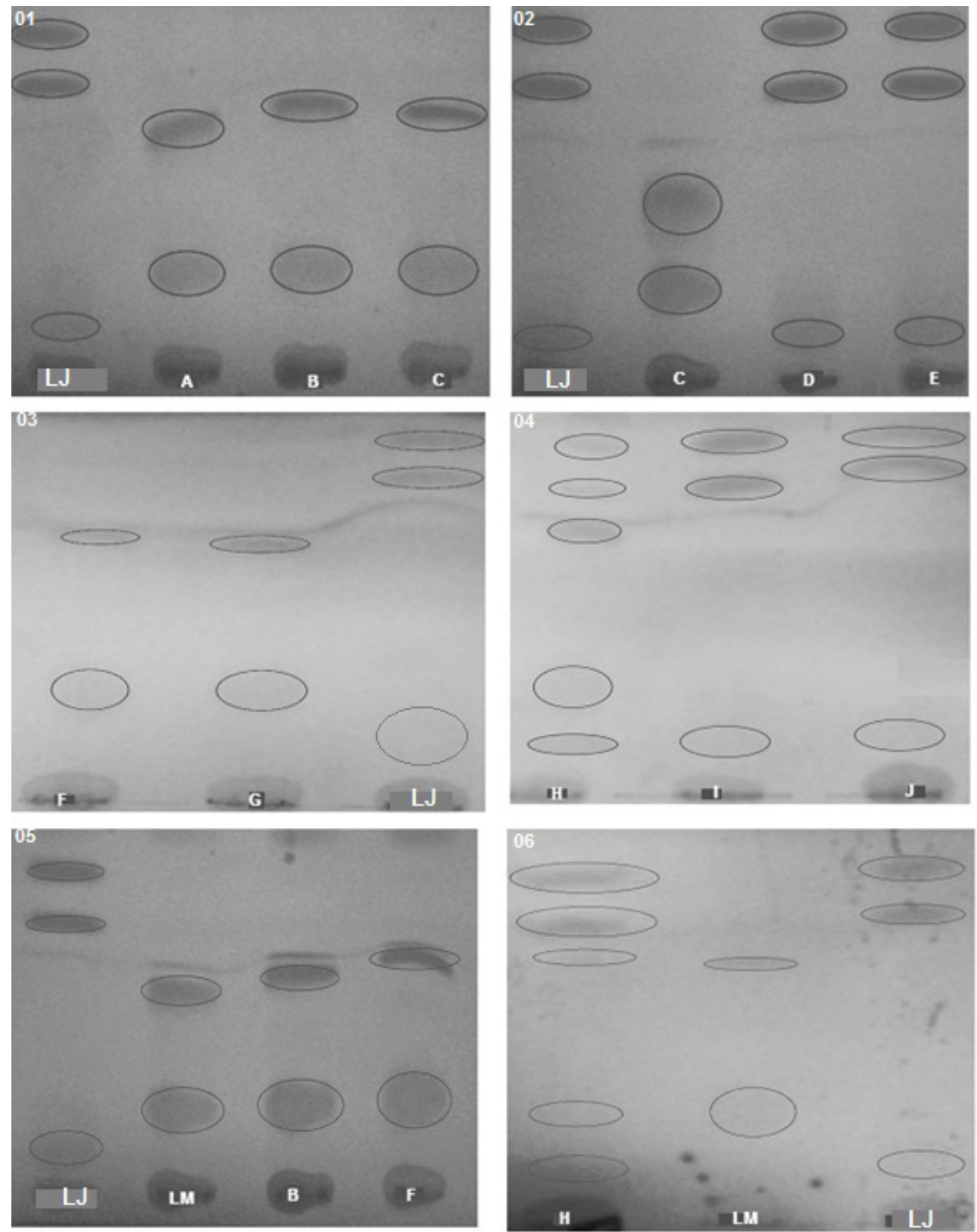

FIGURA 1. Cromatoplacas das amostras comerciais de leite de janaguba, amostra de LJ e látex de mangabeira (Hancornia speciosa; LM).

de E. coli, P. aeruginosa, S. aureus, Salmonella e Gram-negativas bile tolerantes. Os cuidados básicos de higiene durante a extração, como o uso de utensílios limpos (faca, recipiente de coleta e colher), e de água filtrada e fervida na diluição do látex são fundamentais para uma boa qualidade microbiológica do mesmo.

Os resultados obtidos da análise microbiológica de preparações comerciais à base de leite de janaguba revelaram contaminação bacteriana e fúngica em todas as amostras, cerca de $10^{5} \mathrm{UFC} / \mathrm{g}$ para aeróbios totais e fungos.

Considerando que o valor de referência para contagem de fungos e leveduras é de $10^{2}$ UFC/g (mL) (Farmacopeia Brasileira, 2010), a elevada contagem de fungos constitui um risco, em virtude da possibilidade desses serem produtores de micotoxinas. Estas substâncias podem ocasionar 
intoxicações agudas ou crônicas quando da ingestão de produtos contaminados por bolores, mesmo na forma de chá, pois são termoestáveis (Rocha et al., 2004).

O leite de janaguba, pela forma de apresentação (embalagem reaproveitada e rotulagem com ausência do nome de fabricante e CNPJ), possui fabricação artesanal, sendo, pois, um produto passível de várias fontes de contaminação. O próprio processo artesanal de extração já é susceptível à contaminação microbiana, onde é comum o emprego de uma esponja e água na remoção do látex da planta (Linhares \& Pinheiro, 2013). Outro importante meio de contaminação é a qualidade da água utilizada, sendo esta o veículo mais comum em formulações farmacêuticas. Segundo a Organização Mundial de Saúde, $85 \%$ das doenças conhecidas estão relacionadas com água (Barbosa et al., 2009). Na fabricação do leite de janaguba, o látex extraído é diluído em água em uma proporção de 1:4 ou 1:10. A água utilizada tem origem incerta e não se sabe se a mesma passa por algum tipo de tratamento ou descontaminação, o que aumenta a preocupação acerca de sua qualidade (Mariano et al., 2008). O armazenamento desse produto constitui também um importante fator de contaminação. Fitoterápicos sofrem decomposição por bactérias e aumento da temperatura (Matos, 1998); ao serem armazenados em recipientes inadequados podem ser contaminados por fungos, acelerando a decomposição do produto e causando efeitos indesejáveis (Silveira et al., 2008).

Por fim, a determinação das características físico-quimicas e qualidade microbiológica de dez produtos apresentou resultados preocupantes. Há uma grande variabilidade nas referidas características e contaminação microbiana excedente aos valores preconizados pela Farmacopeia Brasileira.

O fato é que, apesar do problema, as pessoas que fazem uso relatam cura com o tratamento (Soares, 2015); ou seja, a elevada carga microbiana não está decompondo os princípios ativos, como também, parece não se tratar de micro-organismos patogênicos. Entretanto, há a necessidade de treinamento para os extrativistas de látex, a fim de que conheçam cuidados básicos para evitar excessiva contaminação microbiana. Em adição, o látex de mangabeira também possui atividades anti-úlcera (Moraes et al., 2008), antiinflamatória (Marinho et al., 2011) e antitumoral in vitro (Ribeiro et al., 2012), o que provavelmente não elimina a credibilidade no uso das preparações rotuladas como leite de janaguba.

Observa-se que há a urgente necessidade da implantação de uma efetiva farmacovigilância de fitoterápicos, a fim de tornar seu consumo mais seguro e racional e, desta forma, reduzir ou eliminar os riscos à população usuária.

\section{REFERÊNCIA}

AGUIAR FILHO, S.P.; BOSCO, J.; ARAÚJO, I.A. A mangabeira (Hancornia speciosa Gomes): domesticação e técnicas de cultivo. 1.ed. João Pessoa: Emepa-PB, 1998. 7p.

AMARO, M.S. et al. Morfologia de frutos, sementes e de plântulas de janaguba (Himatanthus drasticus (Mart.) Plumel - Apocynaceae). Revista Brasileira de Sementes, v. 28, n. 1, p. 63-71, 2006.

BARBOSA, D.A.; LAGE, M.M.; BADARÓ, A.C.L. Qualidade microbiológica da água dos bebedouros de um campus universitário de Ipatinga, Minas Gerais. Revista Digital de Nutrição, v. 3, n. 5, p. 505-517, 2009.

COLARES, A.V. et al. Phytochemical and biological preliminary study of Himatanthus drasticus (Mart.) Plumel (janaguba). Pharmacognosy Magazine, v. 4, n. 14, p. 73-77, 2008a.

COLARES, A.V. et al. Efeito gastroprotetor do látex de Himatanthus drasticus (Mart.) Plumel (Janaguba). Infarma, v. 20, n. 11/12, p. 34-36, 2008b.

FARMACOPEIA BRASILEIRA. v. 1. 5. ed. Brasília, 2010. P. 192-206. Disponível em: <http://www.anvisa.gov.br/ hotsite/cd_farmacopeia/pdf/volume1\%2020110216. pdf>. Acesso 30 mai. 2011.

FORZZA, R.C.; BAUMGRATZ, J.F.A.; BICUDO, C.E.M.; CARVALHO-JUNIOR, A.A.; COSTA, A.; COSTA, D.P.; HOPKINS, M.; LEITMAN, P.M.; LOHMANN, L.G.; MAIA, L.C.; MARTINELLI, G.; MENEZES, M.; MORIM, M.P.; COELHO, M.A.N.; PEIXOTO, A.L.; PIRANI, J.R.; PRADO, J.; QUEIROZ, L.P.; SOUZA, V.C.; STEHMANN, J.R.; SYLVESTRE, L.S.; WALTER, B.M.T.; ZAPPI, D. Catálogo de plantas e fungos do Brasil. v. 1. 1.ed. Rio de Janeiro: Andrea Jakobsson Estúdio/Instituto de Pesquisas Jardim Botânico do Rio de Janeiro, 2010. p. 626.

FREITAS, A.V.L. Recursos genéticos em quintais de comercialização de plantas de uso medicinal no município de São Miguel-RN. 2009. 192p. Dissertação (Mestrado - Área de concentração em Agronomia) Universidade Federal Rural do Semi-árido, Mossoró.

GABRIELS, M.; PLAIZIER-VERCAMMEN, J. Experimental designed optimization and stability evaluation of dry suspensions with artemisini derivatives for paedriatric use. International Journal of Pharmaceutics, v. 283, p. 19-34, 2004.

LACHMAN, L.; LIEBERMAN, H.A.; KANIG, J.L. 1.ed. Teoria e prática na indústria farmacêutica. v. 2. Lisboa: Fundação Calouste Gulbekian, 2001. 500p.

LEITE, F. Validação em análise química. 3. ed. Campinas: Átomo, 1998. 224p.

LEITE, G.O. et al. Gastroprotective effect of medicinal plants from Chapada do Araripe, Brazil. Journal of Young Pharmacy, v. 1, n. 1, p. 54-56, 2009.

LINHARES, J.F.; PINHEIRO, C.U.B. Caracterização do sistema de extração de látex de janaúba (Himatanthus Willd. Ex Schult. - Apocynaceae), no município de Alcântara, estado do Maranhão, Brasil. Revista PanAmazônica de Saúde, v. 4, n. 1, p. 23-31, 2013. 
LORENZI, H.; MATOS, F.J.A. Plantas medicinais no Brasil: nativas e exóticas. 2 ed. Nova Odessa: Plantarun, 2008. 512p.

LUCETTI, D. L. et al. Anti-inflammatory effects and possible mechanism of action of lupeol acetate isolated from Himatanthus drasticus (Mart.) Plumel. Journal of Inflammation, v. 7, n. 60, p. 1-11, 2010.

MARCONDES, N.S.P.; ESMERINO, L.A. Qualidade microbiológica de plantas medicinais cultivadas em hortas domésticas. Publicações Biológicas, v. 16, n. 2, p. 133-138, 2010.

MARIANO, M.B; VIDAL, C.M.D.S; SOUZA, J.B. Avaliação da qualidade microbiológica da água para balneabilidade do Salto Manduri, Prudentópolis - PR. In: SEMANA DE ESTUDOS DA ENGENHARIA AMBIENTAL, 6., 2008, Paraná. Anais. Paraná: DENAM, 2008. p. 1-9.

MARINHO, D.G. et al. The latex obtained from Hancornia speciosa Gomes possesses anti-inflammatory activity. Journal of Ethnopharmacology, v. 135, n. 2, p. 530537, 2011.

MATOS, F.J.A. Farmácias Vivas: sistema de utilização de plantas medicinais projetado para pequenas comunidades. 3. ed. Fortaleza: Editora da UFCE, 1998. 219p.

MODESTO, M.M.L.S. Aspectos ecológicos e socioeconômicos de Himatanthus articulata (Wahl.) Woodson. janaguba da Chapada do Araripe. 1997. 55p. Monografia (Especialização - Área de concentração em Botânica) - Universidade Regional do Cariri, Crato.

MORAES, T.M. et al. Hancornia speciosa: indications of gastroprotective, healing and anti-Helicobacter pylori actions. Journal of Ethnopharmacology, v. 120, n. 2, p. 161-168, 2008.

MOUSINHO, K.C. et al. Efeito antitumoral de proteínas laticíferos de Himatanthus drasticus (Mart.) Plumel Apocynaceae. Journal of Ethnopharmacology, v. 137, n. 1, p. 421-426, 2011.

NASCIMENTO, V.T. et al. Controle de qualidade de produtos a base de plantas medicinais comercializados na cidade de Recife-PE: erva-doce (Pimpinella ansium L.), quebra-pedra (Phyllanthus spp.), espinheira santa (Maytenus ilicifolia Mart.) e camomila (Matricaria recutita L.). Revista Brasileira de Plantas Medicinais, v. 7, n. 3, p.56-64, 2005

OLIVEIRA, S.E.M. et al. Isolation and quantitative HPLC-PDA analysis of lupeol in phytopharmaceutical intermediate products from Vernonanthura ferruginea (Less.) H. Rob. Química Nova, v. 35, n. 5, p. 10411045, 2012.

PEIXOTO, M. Janaguba. Diário do Nordeste, Fortaleza, CE, 26 de setembro de 2001. p. 4.

PINHEIRO, E. et al. O látex e a borracha da mangabeira.
Documentos, v. 202, n. 9702, p. 1-35, 2004. Belém: Embrapa Amazônia Oriental, 2004. Disponível em:<http:// ainfo.cnptia.embrapa.br/digital/bitstream/item/63595/1/ Oriental-Doc202.PDF>. Acesso em: 31 mai. 2011.

PLUMEL, M. M. Repartition geographique du genre Himatanthus em Amerique Tropicale. Comptes Rendus Society of Biogeography, v. 66, n. 3, p. 103-127, 1990.

RIBEIRO, S.S. et al. Evaluation of the cytotoxic activity of some Brazilian medicinal plants. Planta Medica, v. 78, n. 14, p. 1601-1606, 2012.

ROCHA, L.D.O. et al. Análise da contaminação fúngica em amostras de Cassia acutifolia Delile (sene) e Peumus boldus (Molina) Lyons (boldo-do-Chile) comercializadas na cidade de Campinas, Brasil. Revista Brasileira de Ciências Farmacêuticas, v. 40, p. 521-527, 2004.

SAMPAIO, T.S. Estudo fitoquímico de Hancornia speciosa Gomes: isolamento, determinação estrutural e atividade biológica. 2008. 190p. Dissertação (Mestrado - Área de concentração em Química) Universidade Federal de Sergipe, São Cristóvão.

SHOJAEIA, A.; AREFINIA, R. Analysis of the sedimentation process in reactive polymeric suspensions. Chemical Engineering Science, v. 61, p. 7565-7578, 2006.

SILVA, J.R.A. et al. Contribution to the study of Himatanthus sucuuba: látex macromolecule, microfilaments and carbohydrates. Acta Amazônica, v. 33, n. 1, p. 105110, 2003.

SILVEIRA, P.F.D. et al. Farmacovigilância e reações adversas às plantas medicinais e fitoterápicos: uma realidade. Revista Brasileira de Farmacognosia, v. 18, n. 4, p. 618-626, 2008.

SIMÕES, C.O.; CCHENKEL, E.P.; GOSMANN, G.; MELLO, J.C.P.; MENTZ, L.A.; PETROVICK, P.R. Farmacognosia: da planta ao medicamento. 3. ed. Porto Alegre: Ed. da Universidade/ UFRGS, 2001.

SOARES, F.P. Avaliação do potencial farmacoquímico de derivado vegetal oriundo da Chapada do Araripe - Cariri: látex de janaguba (Himatanthus drasticus (Mart.) Plumel). 2015. 182p. Tese (Doutorado - Área de concentração em Desenvolvimento e Inovação Tecnológica de Medicamentos) - Universidade Federal do Ceará, Fortaleza.

SOUSA, E.L. Atividade anti-tumoral de Himatanthus drasticus (Mart.) Plumel-Apocynaceae (janaguba). 2009. 93p. Dissertação (Mestrado-Área de concentração em Ciências Farmacêuticas) - Universidade Federal de Pernambuco, Recife.

SOUZA, F.S.; MACIEL, C.C.S. Produtos fitoterápicos e a necessidade de um controle de qualidade microbiológico. Revista Eletrônica de Ciências, v. 3, n. 2, p. 22-30, 2010. 